\title{
Addressing Social Needs of the Patients by Physicians and Nongovernmental Organizations in Iran
}

\author{
Mohammad Zakaria Pezeshki ${ }^{1,}$ \\ ${ }^{1}$ Department of Community\& Family Medicine and Social Determinants of Health Research Center, Tabriz University of Medical Sciences \\ ${ }^{*}$ Corresponding author: Mohammad Zakaria Pezeshki, Department of Community\& Family Medicine and Social Determinants of Health Research Center, Tabriz University of Medi- \\ cal Sciences, Tabriz, Iran. E-mail: zakaria.pezeshki@gmail.com
}

Received: 22 Sep 2016

Accepted: 21 Nov 2016

Ppub: 23 Dec 2016

\begin{abstract}
Background: There are several researches showing that "social determinants of health" are associated with the clinical outcome of the patients. Four out of 5 American primary care physicians do believe that addressing social needs of their patients are as important as addressing medical conditions of their patients. Unfortunately there are few researches on the feasibility of addressing social needs of the patients by physicians and nongovernmental organizations (NGOs).

Methods: Recent research initiative of family physicians in New Mexico, USA about the feasibility of assessing and treating social needs of the patients resulted in state level policy making for addressing social needs of the patients. In this presentation I review three initiatives in USA, two of them by physicians and one of them by a NGO that address the social needs of the patients. The questionnaires that are used for measuring social needs in these initiatives will be introduced.

Results: I will discuss how designing a collaborative initiative between Social Deputy of Iranian Ministry of health, NGOs and Health Promoting Hospitals in Iran may improve patients outcomes by better addressing their social needs.

Conclusions: The role of Iranian family physicians in identifying and addressing the social needs of the patients in primary care clinics with be explained.

Keywords: Social Determinants of Health; Family Physicians; Nongovernmental Organizations; Ministry of Health
\end{abstract}

